\title{
Bronchial Embolization with an Endobronchial Watanabe Spigot for Broncho-Gastric Tube Fistula
}

\author{
Terunaga Inage ${ }^{a}$ Takahiro Nakajima ${ }^{a}$ Taiki Fujiwara ${ }^{a}$ Kentaro Murakami ${ }^{b}$ \\ Masaya Uesato $^{b}$ Hisahiro Matsubarab ${ }^{b}$ Ichiro Yoshino ${ }^{a}$ \\ Departments of a General Thoracic Surgery and ${ }^{b}$ Frontier Surgery, Chiba University Graduate School of \\ Medicine, Chiba, Japan
}

\section{Established Facts}

- Broncho-gastric tube fistula as a complication after esophagectomy for esophageal cancer can cause refractory pneumonia, often resulting in respiratory failure and life-threatening conditions.

\section{Novel Insights}

- Endobronchial Watanabe spigot placement was effective for the management of a case of bronchogastric tube fistula.

\section{Keywords}

Broncho-gastric tube fistula · Endobronchial Watanabe spigot

\section{Abstract}

Anastomotic failure of a gastric tube inserted for reconstruction following esophagectomy, which is relatively rare, causes pleural infection and persistent pleural irritation, leading to communication with the pulmonary parenchyma. Although several interventions have been reported to treat such broncho-gastric tube fistulas, refractory cases remain. We herein report the successful treatment by endo-

\section{KARGER}

(C) 2017 S. Karger AG, Basel

E-Mail karger@karger.com

www.karger.com/res scopic bronchial occlusion with an endobronchial Watanabe spigot in 2 patients who suffered from the above complication.

(c) 2017 S. Karger AG, Basel

\section{Introduction}

Broncho-gastric tube fistula is a rare complication after esophagectomy for esophageal cancer. Fistulas can cause refractory pneumonia, often resulting in respiratory failure and life-threatening conditions [1]. The endobronchial Watanabe spigot (EWS) was first intro-

Takahiro Nakajima, MD, PhD

Department of General Thoracic Surgery Chiba University Graduate School of Medicine

1-8-1 Inohana, Chuo-ku, Chiba 260-8670 (Japan)

E-Mail takahiro_nakajima@ med.miyazaki-u.ac.jp 

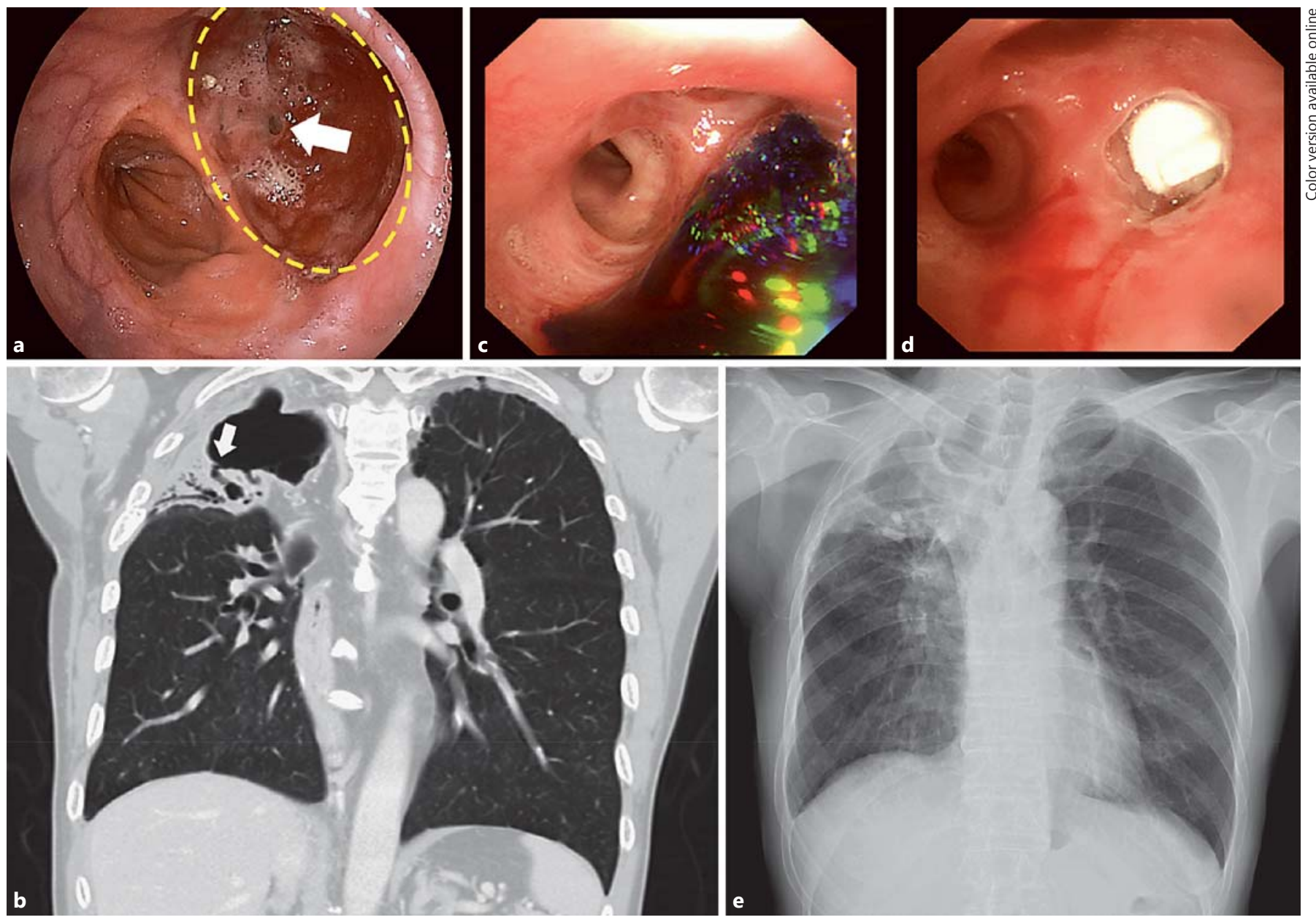

Fig. 1. Imaging findings for case 1. a Endoscopy via the gastric tube showing the fistula (arrow) and cavity of the right upper lobe (circle). b Chest computed tomography showing cavity formation in the right upper lobe and a fistula between the gastric tube and the

right upper lobe of the lung (arrow). c Indigo carmine was drained from the right posterior segmental bronchus (B2a). d A total of 3 EWSs were successfully placed at the referring bronchus. e After EWS placement, the aspiration pneumonia was relieved.

duced as an endobronchial occlusion silastic spigot for persistent pneumothorax [2]. The spigot can also be used for bronchopleural fistula as well as temporary stabilization for hemostasis by occluding the airway [3]. The EWS is well-designed for smooth insertion into the bronchial lumen and has studs on the surface to prevent migration.

We herein report 2 cases of broncho-gastric tube fistula after transthoracic esophagectomy for esophageal cancer that were successfully treated by endoscopic bronchial occlusion with placement of an EWS.

\section{Case Report}

Case 1

A 60-year-old man was suffering from pneumothorax and pleural infection of the right side due to dehiscence of gastric tube anastomosis after esophagectomy for esophageal cancer. Repeated thoracotomies for direct suture and muscle plombage were unsuccessful. Transesophageal intervention by endoscopy also failed to occlude the fistula (Fig. 1a). The patient had not been able to take anything orally for 7 years, and repeated pneumonia due to saliva aspiration had gradually deteriorated his respiratory function. Chest computed tomography showed a fistula between the gastric tube and the right upper lobe of the lung (Fig. 1b). A fluoroscope revealed extravasation of barium from the gastric tube into the right upper lobe of the lung. We therefore considered performing endoscopic bronchial occlusion. To locate the referring bronchus, $5 \mathrm{~mL}$ of indigo carmine was injected into the gastric tube. Bron- 

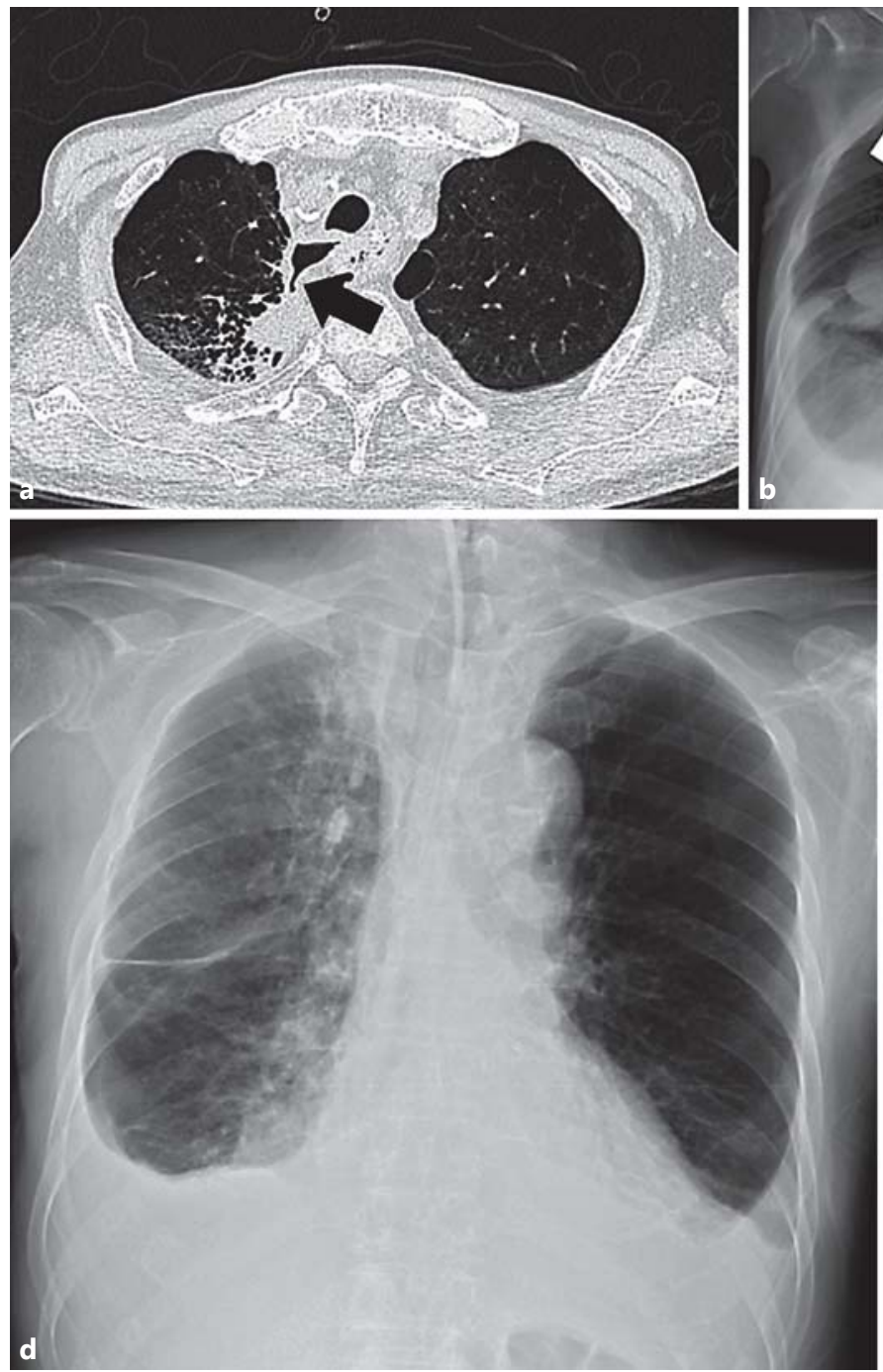

Fig. 2. Imaging findings for case 2 . a A fistula was detected on chest computed tomography (arrow). b Extravasation of barium from the gastric tube was observed (arrow). c Indigo carmine with pus was drained from the apical segmental bronchus (right B1a).d Im-

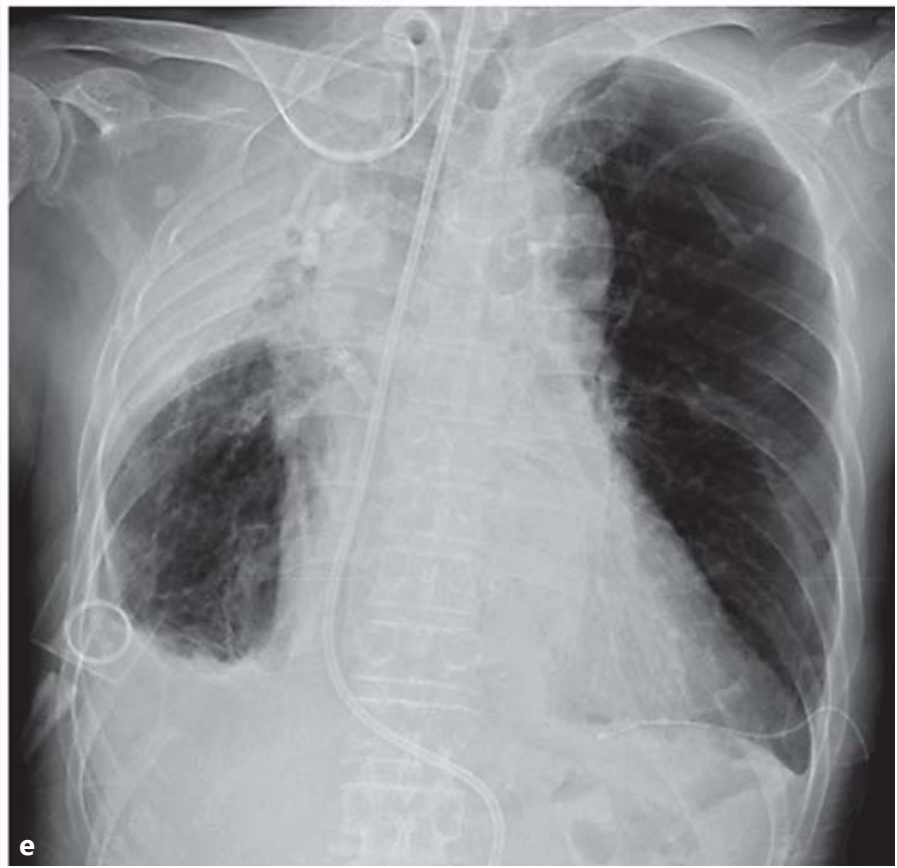

mediately after the placement of the EWSs, the patient's pneumonia was improved. e At 20 days after EWS insertion, chest X-ray showed right upper lobe atelectasis.

\section{Case 2}

A 70-year-old man underwent chemoradiotherapy followed by esophagectomy for esophageal cancer. Although the early postoperative course was uneventful, he developed pneumonia at 56 days after surgery. A fistula between the gastric tube and right upper lobe of the lung was suspected based on chest computed tomography (Fig. 2a). Fluoroscopy showed extravasation of barium from the gastric tube into the right upper lobe bronchus (Fig. 2b). Bronchoscopy revealed drainage of indigo carmine that had been injected into the gastric tube from the subsegmental branch of the right apical segmental bronchus (B1a) (Fig. 2c). One 6-mm-sized and one 7 -mm-sized EWS were placed at B1a and B1, respectively. Immediately after the procedure, the indigo carmine influx was found to have been stopped based on a confirmatory injection 
(Fig. 2d). Right upper lobe atelectasis was found on chest X-ray (20 days after the procedure); however, the patient was relieved of repeated pneumonia, and his general condition recovered (Fig. 2e). He was transferred to a rehabilitation hospital 165 days after the procedure. During follow-up, he developed recurrence of esophageal cancer. He was transferred to another hospital and died due to cancer progression 7 months after the procedure.

\section{Bronchial Embolization Using the EWS}

The patients were intubated using a \#8.0 endotracheal tube under local anesthesia with moderate conscious sedation. Local anesthetic (lidocaine) was applied to the pharynx by nebulizer and pharyngeal spray initially. Moderate conscious sedation was achieved using 1-2 $\mathrm{mg}$ of midazolam before starting the procedure. Bolus ( $2 \mathrm{~mL}$ ) 1\% of lidocaine was given through the instrument channel and an additional $1 \mathrm{mg}$ of midazolam was given depending on the level of conscious sedation during the procedure. Conventional bronchovideoscope (BF TYPE 260; Olympus, Tokyo, Japan), which has a $4.9-\mathrm{mm}$ outer diameter with a $2.0-\mathrm{mm}$ instrument channel, was used for this procedure. First, $20 \mathrm{~mL}$ of $0.1 \%$ indigo carmine solution (the same concentration as for upper gastrointestinal use) was given though the gastric tube to identify the targeted bronchus. When the targeted bronchus was identified, EWSs were inserted using conventional biopsy forceps (FB-19C-1, Olympus) following the same procedure as previously described [4]. A 5- or 6-mm EWS was selected to occlude subsegmental bronchus, and a 7-mm EWS was used for segmental bronchus occlusion.

\section{Discussion}

Broncho-gastric tube fistulas are rarely observed as a complication of esophagectomy for esophageal cancer. Treatment is often difficult, and some cases are refractory to aggressive surgery as well as conservative interventions. We herein reported 2 cases of broncho-gastric tube fistula, the first of which was refractory to multiple attempts to close the fistula. After the failure of surgical and endoscopic interventions, endobronchial occlusion of the bronchus referring to the fistula using an EWS successfully managed the complication and allowed the patient to recover from repeated respiratory infections. For the second patient, this same procedure was chosen at the outset.

Endobronchial occlusion was initially developed to treat patients with persistent air leakage from the lung due to broncho-pleural fistula as well as ordinary pneumothorax. Various types of occlusion material have been invented, including Oxycel-cotton, Bismuth Tribromophenate (Xeroform) [5], fibrin glue [6], gelatin sponge, and cyanoacrylate. Each material has advantages and disadvantages, but one major factor limiting the utility of these materials for endobronchial occlusion is the diffi- culty in achieving long-term placement with a stable condition. Most of these materials are absorbable and were not developed for endobronchial occlusion; therefore, they migrate easily. The EWS, which was specifically designed for endobronchial occlusion, was first introduced in 1991 by Watanabe et al. [7]. The EWS is a nonabsorbable, silastic, spindle-shaped plug with studs on the surface to prevent migration. EWS can be inserted safely, even in patients with a severe general condition $[4,8]$, because it can be placed using a flexible bronchoscope. Bronchial occlusion with an EWS can also be used for temporary control of massive hemoptysis.

In the present report of a case of broncho-gastric tube fistula, pulmonary infection due to saliva leakage from the gastric tube was managed by endobronchial airway occlusion using an EWS. After the procedure, the patient's general condition recovered, and oral intake was resumed. Extremely few cases of broncho-gastric tube fistula have been reported, and despite a thorough search using Pubmed and Google Scholar (accessed on December 28, 2016), to our knowledge, this is the first report of an EWS being used for the treatment of broncho-gastric tube fistula. Due to the small number of publications on long-term EWS placement, the long-term outcome of EWS is unknown. In our experience, the longest period of EWS placement was more than 3 years and the patient was in a stable condition without any lung tissue response or injury.

In conclusion, endobronchial EWS placement was effective for the management of a case of broncho-gastric tube fistula.

\section{Acknowledgements}

The authors thank the Japan Medical Communication for reviewing the English manuscript. No financial support was obtained for this study.

\section{Financial Disclosure and Conflicts of Interest}

Takahiro Nakajima received an unrestricted grant for medical research from Harada Corporation by participating in post-marketing surveillance. The sponsor of the study had no role in the study design, conduct of the study, data collection, data management and interpretation, preparation of the report, review of the report, or approval of this report. The other authors have no conflicts of interest to disclose.
$378 \quad$ Respiration 2017;94:375-379
Inage/Nakajima/Fujiwara/Murakami/ Uesato/Matsubara/Yoshino 


\section{Author Contributions}

Dr. Nakajima had full access to all of the data in the study and takes responsibility for the integrity of the data and the accuracy of the data analysis. Dr. Inage collected the data and wrote and revised the manuscript. Dr. Fujiwara, Dr. Murakami, Dr. Uesato, and Dr. Matsubara contributed to data acquisition, analysis, and interpretation. Dr. Yoshino contributed to study design, data analysis and interpretation. T.I., T.N., T.F., K.M., M.U., H.M., and I.Y. contributed to the drafting, revising, and review of the manuscript for important intellectual content and final approval of the version to be published.

\section{References}

1 Osaki T, Matsuura H: Thoracic empyema and lung abscess resulting from gastropulmonary fistula as a complication of esophagectomy. Ann Thorac Cardiovasc Surg 2008; $14: 172$ 174.

2 Watanabe Y, Matsuo K, Tamaoki A, et al: Bronchial occlusion with endobronchial Watanabe spigot. J Bronchol 2003;10:264267.

3 Dutau H, Palot A, Haas A, et al: Endobronchial embolization with a silicone spigot as a temporary treatment for massive hemoptysis. Respiration 2006;73:830-832.
4 Inage $\mathrm{T}$, Nakajima $\mathrm{T}$, Wada $\mathrm{H}$, et al: Endobronchial embolization using a Watanabe spigot for persistent air leakage. Emerg Med Health Care 2016;4:1.

5 Fujiwara M, Sassoon S, Kota C, et al: Successful closure of bronchopleural fistula with Xeroform dressing. J Bronchology Interv Pulmonol 2012;19:251-254.

6 Ishikawa K, Kato T, Aragaki M, et al: Endobronchial closure of a bronchopleural fistula using a fibrin glue-coated collagen patch and fibrin glue. Ann Thorac Cardiovasc Surg 2013;19:423-427.
7 Watanabe Y, Hiraki S, Araki M: Bronchial embolization using dental impressin material in a case of pyelo-bronchial fistula with candida fungemia (in Japanese with English abstract). J Jpn Soc Bronchol 1991;13:607-610.

8 Sasada S, Tamura K, Chang YS, et al: Clinical evaluation of endoscopic bronchial occlusion with silicone spigots for the management of persistent pulmonary air leaks. Intern Med 2011;50:1169-1173. 